\title{
EVALUACIÓN DEL DOCENTE UNIVERSITARIO Y NECESIDADES DE RECONOCIMIENTO: ANÁLISIS Y RELACIÓN
}

\author{
EVALUATION OF THE UNIVERSITY \\ PROFESSOR AND RECOGNITION \\ NEEDS: ANALYSIS AND RELATIONSHIP
}

Luciano Carlos Andrés Scattolon Huapaya lucianoscattolonh@unife.edu.pe

\section{RESUMEN}

La investigación se llevó a cabo con el objetivo de analizar la relación entre el sistema de evaluación del docente universitario y su satisfacción de necesidades de reconocimiento. Es un estudio inductivo no experimental, de tipo descriptivo y correlacional de enfoque mixto. La muestra quedó constituida por un total de 28 docentes del área de inglés de la sede central de una universidad privada de Lima, en el ciclo 2018-II, utilizándose un muestreo probabilístico por conveniencia. Se utilizó el cuestionario como instrumento y la técnica de encuesta. El sistema utilizado por la universidad muestra evidencia de relacionarse positivamente con las necesidades de reconocimiento del docente universitario; se ha contribuido con la constatación de procesos y herramientas aplicadas a la evaluación y el impacto en el colaborador, específicamente en su satisfacción de necesidades, orientado a generar un valor agregado para el ofrecimiento del servicio educativo que representa un diferencial competitivo en el contexto global actual.

PALABRAS CLAVE

Profesor universitario, satisfacción laboral, enseñanza superior, evaluación docente, reconocimiento.

\section{ABSTRACT}

This research was carried out with the objective of analyzing the relationship between the evaluation of the university professor and the satisfaction of recognition needs. It is a nonexperimental inductive study, of a descriptive and correlational type of mixed approach. The sample consisted of a total of 28 professors from the English area of the main campus of a Private University of Lima, in the 2018-II cycle, using a probabilistic sampling for convenience. The questionnaire was conducted as an instrument and the survey as a technique. The system used by the university shows evidence of relating positively to the recognition needs of the university teacher; the study has contributed with the verification of processes and tools applied to the evaluation and the impact on the collaborator specifically in their satisfaction of needs, oriented to generate an added value for the educational service offer that represents a competitive differential in the current global context.

\section{KEYWORDS}

University professor, job satisfaction, higher education, teacher evaluation, recognition.

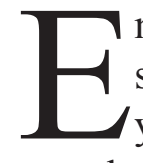
n la actualidad, las universidades han sido influenciadas por la competitividad y el cambio tecnológico conllevado por la globalización. En tal sentido, estas instituciones del ámbito privado deben asegurar su permanencia en el mercado y capacidad de mantener satisfecho a su cliente externo e interno. La gestión eficiente reconoce que tanto los alumnos como los docentes, son vitales en este contexto, el cual requiere crear estrategias de generación de valor y diferenciación por las partes involucradas, como menciona BerryLeonard (1981).

Se realizó esta investigación porque es importante analizar los elementos que comprenden las funciones del docente y cómo impacta en su satisfacción. Se entiende que el docente universitario debe estar motivado para realizar sus funciones eficientemente y orientado hacia la consecución de los objetivos institucionales, por lo que la delimitación de un perfil motivado es de vital importancia, como señala M. Ruiz (2012); además, la universidad debe evaluar cómo lo realiza, para poder orientar sus esfuerzos hacia una calidad medible y mejorable a través de prácticas continuas que impacten positivamente en la comunidad universitaria, como mencionan Loureiro \& Míguez (2016) y conocer las necesidades del 
docente para poder ajustar los criterios con los cuales son evaluados.

En tal sentido, se llevó a cabo esta investigación para analizar el nivel de satisfacción de las necesidades de reconocimiento del docente universitario y elevar propuestas de mejora y recomendaciones a las autoridades académicas pertinentes al representar importancia como problema no profundizado. En este contexto, se limitó a los profesores de inglés en la muestra comprendida del ciclo de marzo a julio del 2018. Además, se ha sugerido profundizar en investigaciones relacionadas con la formación profesional y desarrollo en el sector educación superior. También por su viabilidad a nivel personal y económico.

Esta investigación permite ser replicada por la comunidad académica a través de sistemas integrados, como mencionan García Cabrero, Loredo Enríquez \& Carranza Peña (2008), que comprendan el procedimiento para evaluar al docente utilizado por la universidad y por otro lado, por las necesidades de reconocimiento del docente; además, se podrá recoger información que permita ser interpretada por la gestión estratégica del cuerpo docente y tomar acciones correctivas o de mejora en un contexto de tiempo y lugar específico y ahondar en los diferentes criterios de evaluación adoptados por la institución, como menciona Santos Del Real (2012).

Lo mencionado anteriormente conlleva a realizar la siguiente pregunta: ¿Qué relación existe entre el sistema de evaluación docente y la satisfacción de necesidades de reconocimiento del profesor universitario del área de inglés en la sede central de una universidad privada de Lima, en el ciclo 2018-II?

En respuesta, se han planteado los objetivos específicos, los cuales buscan determinar la relación entre la evaluación docente basada en los aspectos de la izquierda de la imagen, con las necesidades desagregadas con los aspectos de la derecha, para una mejor comprensión.

\section{Figura 1}

Dimensiones del sistema de evaluación y su relación con necesidades de reconocimiento

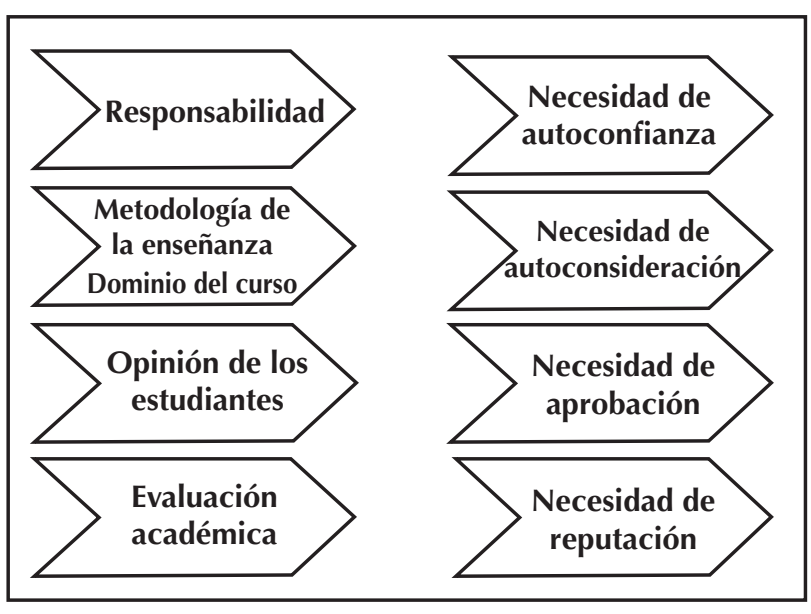

Fuente: Elaboración propia.

\section{Método}

Esta investigación ha permitido generar conocimiento profundo de tercer nivel, aplicándose la correlación entre el sistema de evaluación del docente universitario y la satisfacción de necesidades de reconocimiento.

Se aplicó en la sede central de una universidad de Lima en el ciclo que comprende de marzo a julio del 2018.

Según Sabino (1986), es de tipo descriptivo correlacional, cuya característica es interpretar la realidad de los hechos y descubrir características fundamentales en fenómenos homogéneos a través de criterios sistemáticos que permitan mostrar su comportamiento y estructura y ser descritos, también mencionado por Hernández Sampieri, Fernández y Baptista (2014). El análisis mide la relación entre ambas variables, y expresa cómo se aplicaron las técnicas utilizadas para la recolección de datos, según Hurtado de Barrera (2008).

Se seleccionó como instrumento al cuestionario, debido a su aplicabilidad y factibilidad para medir de manera anónima las dimensiones planteadas. Como técnica se utilizó la encuesta digital, considerándose 32 preguntas que 
comprenden ambas variables con categorías de respuestas según la escala de Likert, del 1 al 5. Se realizó la validación por expertos en educación y psicopedagogía.

Las ventajas que se encontraron fueron las siguientes: a nivel tiempo, cada docente pudo desarrollarla con tranquilidad cuando tenía la disposición para hacerla. Y espacio, pues no requerían estar todos al mismo momento. De esta manera, se consideraron también facilidades económicas y practicidad para obtener los resultados de manera expedita, y mantener el anonimato y discreción correspondiente en las respuestas de los evaluados, tal como lo realizaron Hortigüela Alcalá, Ausín Villaverde, Delgado Benito \& Abella García (2017). La población se constituyó por 30 docentes de la institución, dedicados al área de inglés y se elaboró la muestra con los siguientes datos:

\section{Tabla 1}

Datos para elaboración de la muestra

\begin{tabular}{lll}
\hline $\mathbf{n}$ & Tamaño de la muestra & $\mathbf{x}$ \\
\hline $\mathrm{z}$ & Nivel de confianza & 1.95 \\
$\mathrm{p}$ & Variabilidad positivo & 0.5 \\
$\mathrm{q}$ & Variabilidad negativo & 0.5 \\
$\mathrm{~N}$ & Tamaño de la población & 30 \\
$\mathrm{E}$ & Precisión o el error & 0.05 \\
\hline
\end{tabular}

Fuente: Elaboración propia.

Luego de aplicar el muestreo probabilístico correspondiente, se seleccionó una muestra conformada por 28 docentes, la cual fue accesible para la investigación. Se aplicó la siguiente fórmula estadística, la misma que también fue utilizada por H. Arkin \& Roosevelt Colton, R. (2018).
Fórmula:

$$
\begin{gathered}
n=\frac{z^{2} p q N}{E^{2}(N-1)+Z^{2} p q} \\
n=\frac{\left.(1.95)^{2}(0.5)(0.5) * 30\right)}{(0.05)^{2}(30-1)+(1.95)^{2}(0.5)(0.5)}
\end{gathered}
$$

$n=28$ Tamaño de la muestra total

Cabe indicar que el error de la muestra es de $+/-5 \%$

Luego de la validación por especialistas, se aplicó un formato de prueba en otro grupo docente a fin de obtener una validación complementaria.

Se recibieron apreciaciones y mejoras para el entendimiento del instrumento; tales como, la palabra autónomo por independiente, manera profunda por no superficial, solvente por útiles.

Las dimensiones del sistema de evaluación utilizado por la institución han permitido interpretar y generar una relación con las variables de reconocimiento, además de crear un modelo propio basado en las teorías de Maslow, Mc Clelland y Alderfer.

Dentro de las diferentes necesidades, Maslow (1991) hace un balance entre el aprecio propio y externo. Se consideró a la confianza en uno mismo, la consideración, la aprobación y la reputación.

Además, Mc Clelland (1961) menciona la orientación del individuo hacia la influencia en los demás, el logro a alcanzar metas, y la afiliación para con las personas que lo rodean.

Por último, Alderfer (1969) menciona su modelo en las necesidades de crecer, existir, tener reputación y relacionarse. 
Tabla 2

Modelo Integrado de Teorías de Necesidades

ORIENTACIÓN

INFLUENCIA

LOGRO

AFILIACIÓN

IMPULSO

CRECIMIENTO

Uno se siente

Valorado

Respetado

Desarrollado

NECESIDADES DE RECONOCIMIENTO

Necesidad de

consideración

\section{EXISTENCIA}

Competente

Autónomo

Autoconsciente

Necesidad de

autoconfianza
REPUTACIÓN

RELACIÓN

Destacado

Admirado

recompensado

Aceptado

Efectivo

Perfectible
Necesidad de reputación
Necesidad de aprobación

Fuente: Elaboración propia.

El instrumento presentó 32 afirmaciones sobre el grado de conformidad de ambas variables relacionadas, basado en el sistema de evaluación de la institución para contextualizar al docente y que tenga el conocimiento previo necesario para poder aplicarlo con eficiencia. Esta aplicación se ha dado de manera anónima, a través de la escala de Likert, el docente seleccionó el estar en desacuerdo plenamente o plenamente de acuerdo, para ser procesado.

\section{Procedimiento}

Para poder levantar la información se siguieron una serie de pasos que se detallarán a continuación: en primer lugar, se utilizó la plataforma digital Google Forms con las preguntas y opciones correspondientes; luego, se distribuyó por correo electrónico a los docentes del grupo muestra, para que en su propio tiempo y disponibilidad pudieran completarlo. Para incentivarlos se ofreció una tarjeta de regalo a través de un sorteo aleatorio. También se validó, se registró y se procesó la información a través de Microsoft Excel, por medio de diagramas de Pareto para su interpretación.

\section{Resultados}

\section{Responsabilidad}

Los docentes reconocen los siguientes indicadores como eje principal para su práctica docente y la manera como la universidad los reconoce, los satisface. El estar relacionado de manera positiva impacta en la ejecución de esas tareas de responsabilidad. 


\section{Figura 2}

Resultados sobre la dimensión de responsabilidad

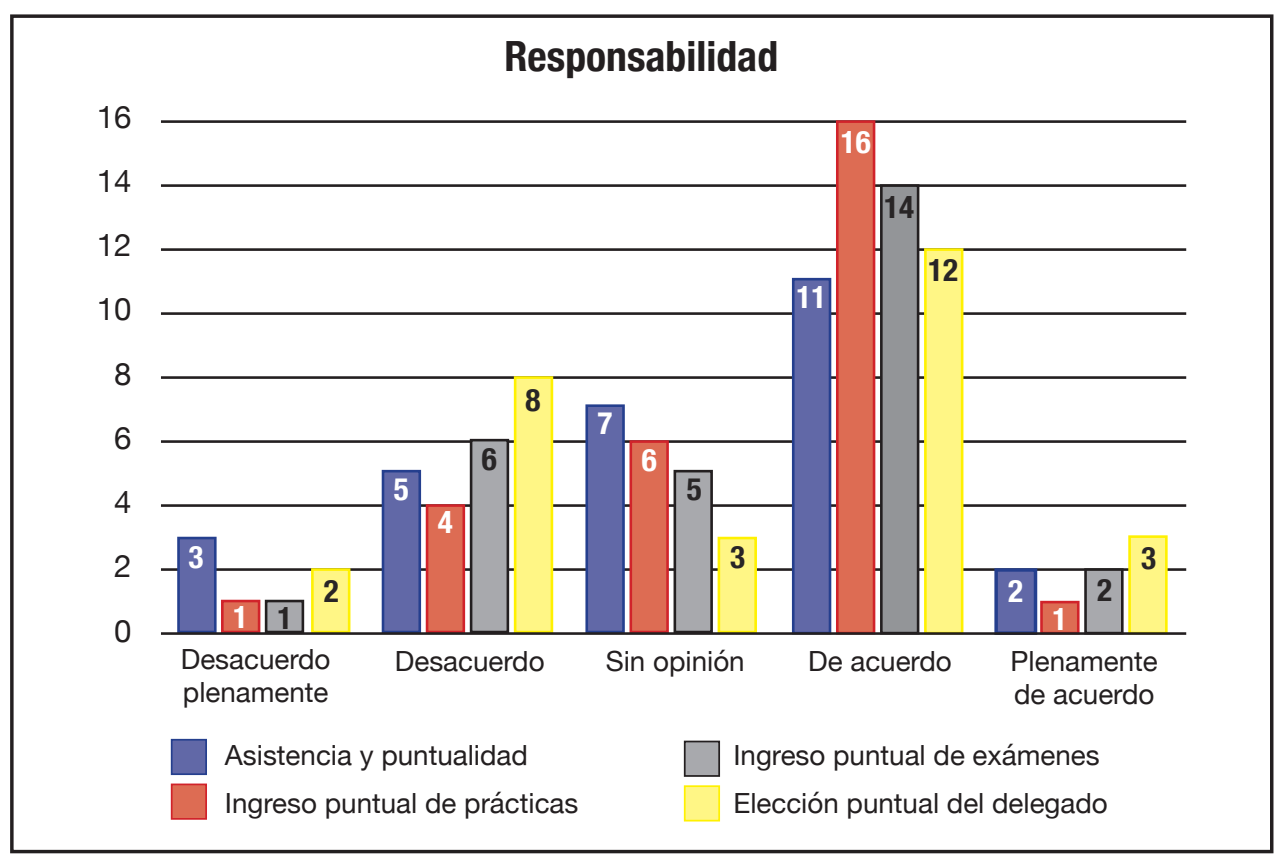

Fuente: Elaboración propia.

La universidad reconoce al docente con el indicador de asistencia y puntualidad y el $39.3 \%$ de la muestra indicó estar de acuerdo sobre sentirse satisfechos. Para el docente, realizar todas sus clases programadas y llegar a tiempo es importante y percibe que la universidad reconoce tal esfuerzo.

Por su parte, la universidad reconoce al docente con el indicador de ingreso puntual de notas de prácticas y el $57.1 \%$ de la muestra indicó estar de acuerdo sobre sentirse satisfechos.

Asimismo, la universidad reconoce al docente con el indicador de ingreso puntual de notas y devolución de exámenes y el $50 \%$ de la muestra indicó estar de acuerdo sobre sentirse satisfechos. En tal sentido, una vez corregidos los exámenes y prácticas, el docente deberá registrar las notas, este indicador también es considerado en su evaluación como elemento para evaluar el reconocimiento que recibe de su centro de labores.

Igualmente, la universidad reconoce al docente con el indicador de elección puntual del delegado de clase y el $42.9 \%$ de la muestra indicó estar de acuerdo, lo que representa un nivel de comunicación de suma importancia entre el docente y el alumnado en general.

\section{Necesidad de autoconfianza}

El docente necesita estar satisfecho en relación a sentir que es competente en su curso, y que conoce sus aspectos fuertes, además que puede desarrollarse bajo el principio de libertad de cátedra. 


\section{Figura 3}

Resultados sobre la dimensión de necesidad de autoconfianza

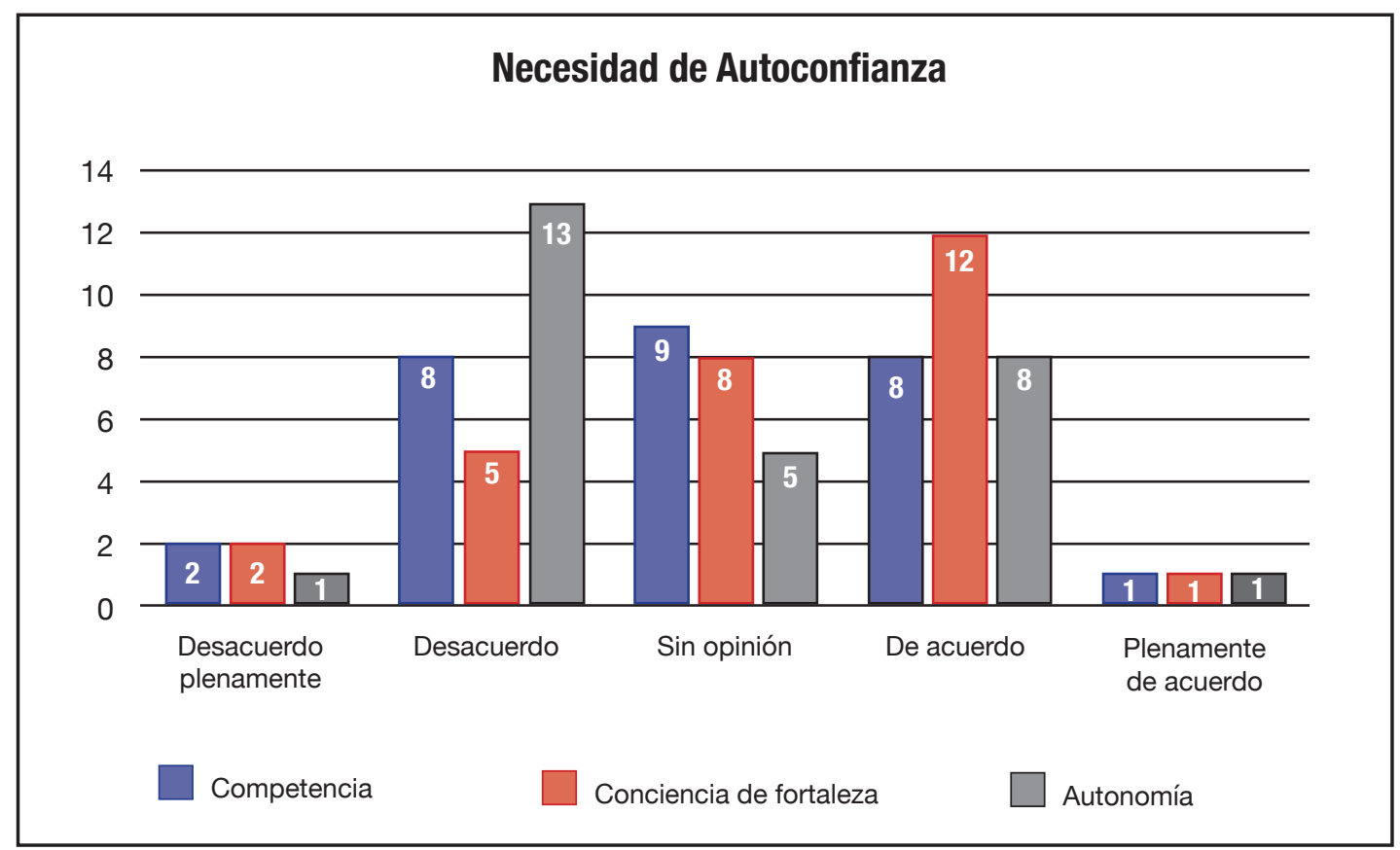

Fuente: Elaboración propia.

La universidad reconoce al docente sobre la dimensión de responsabilidad; en tal sentido, el $32.1 \%$ de la muestra no se siente en desacuerdo ni de acuerdo sobre sentirse competente. Lo que resulta en una opinión neutral sobre el sistema de evaluación.

El $42.9 \%$ de la muestra indicó estar de acuerdo sobre sentirse consciente de sus fortalezas. Por lo que se reconoce que el sistema sí le permite saber los aspectos de buen desarrollo y en los que falta mejorar y en los tiempos en los que ejecuta sus acciones de control.

El $46.4 \%$ de la muestra indicó estar en desacuerdo sobre sentirse autónomo, en relación a seguir una serie de parámetros e indicadores que le quitan libertad en su cátedra. Se puede complementar estudios en este aspecto y abrir nuevas líneas de investigación.
Existe una limitación de criterios bajo el sistema mencionado. El ser puntual en el inicio de sesión puede impactar negativamente en el resto de alumnos que suelen llegar a su sesión y perderse los momentos iniciales. Por otro lado, el ingreso de notas es impactado por eventos o inasistencias ajenas a la voluntad docente, que en el programa o diseño del sistema no se contempla. Además, la elección del delegado se convierte en el cumplimiento del requisito, más allá del valor y responsabilidad que este genera.

\section{Metodología de la enseñanza}

El criterio metodológico de la universidad requiere de un momento inicial, descubrimiento de la utilidad para el estudiante, un espacio para transformar lo aprendido o practicarlo y un cierre definido. 


\section{Figura 4}

Resultados sobre la dimensión de metodología de la enseñanza

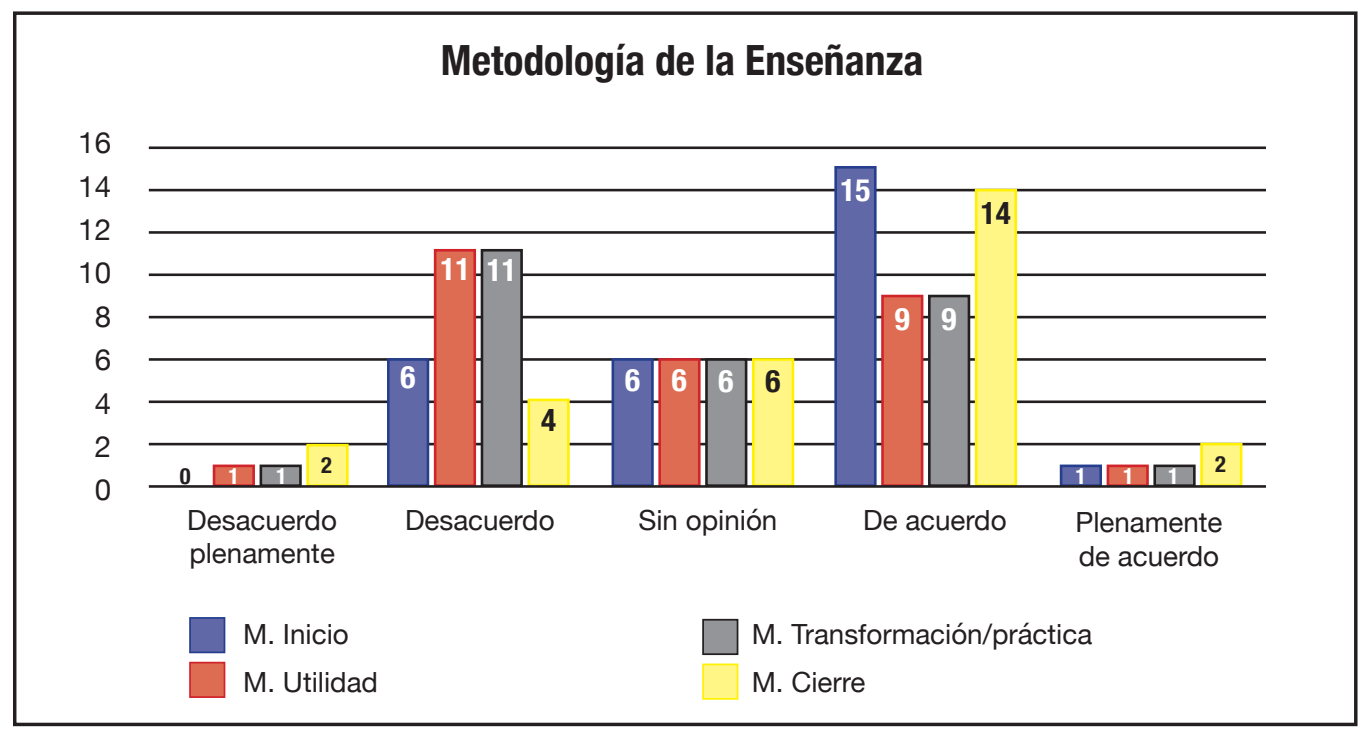

Fuente: Elaboración propia.

La universidad reconoce al docente con el indicador de Momento de Inicio y el 53.6\% de los docentes encuestados indicó estar de acuerdo sobre sentirse satisfechos. Este momento sirve para que el alumno se concentre en la clase.

La universidad reconoce al docente con el indicador de Momento de Utilidad y el 39.3\% de los docentes encuestados indicó estar en desacuerdo sobre sentirse satisfechos. Sirve para activar conocimientos anteriores, despejar dudas y saber qué importancia tiene el tema en contexto.

La universidad reconoce al docente con el indicador de Momento de Transformación y Práctica, y el 39.3\% de los docentes encuestados indicó estar en desacuerdo sobre sentirse satisfechos. Este momento se relaciona con la ejecución en situaciones aplicadas y creación en escenarios relacionados a la vida cotidiana.

La universidad reconoce al docente con el indicador de Momento de Cierre y Práctica, y el $50 \%$ de los docentes encuestados indicó estar de acuerdo sobre sentirse satisfechos, lo cual implica reflexionar sobre lo aprendido y consolidar los aspectos más importantes de las sesiones.
Bajo estos resultados, se desprende que los momentos de inicio y cierre sí satisfacen al docente en la manera del impacto en su evaluación; no obstante, la utilidad y transformación/práctica no tienen el mismo porcentaje de resultados.

Se podría analizar los criterios utilizados en estos dos últimos indicadores y profundizar estos aspectos metodológicos y su impacto en el docente; así como lo que se espera de él en un momento determinado.

El uso de rúbrica como instrumento de medición podría ser revisado y el docente sensibilizado y capacitado para que se pueda estandarizar los criterios de calidad y ver en qué medida algunas actividades pueden variar la ponderación del docente, ya que se necesita orientar la capacitación docente hacia la mejora continua para elevar la calidad de enseñanza, como menciona R. Piña (2010).

\section{Dominio del curso}

El sistema de evaluación del docente incluye el conocimiento de su profesión y que el manejo de los temas sea el adecuado, por lo que se evalúan los siguientes indicadores: 


\section{Figura 5}

Resultados sobre la dimensión de dominio del curso

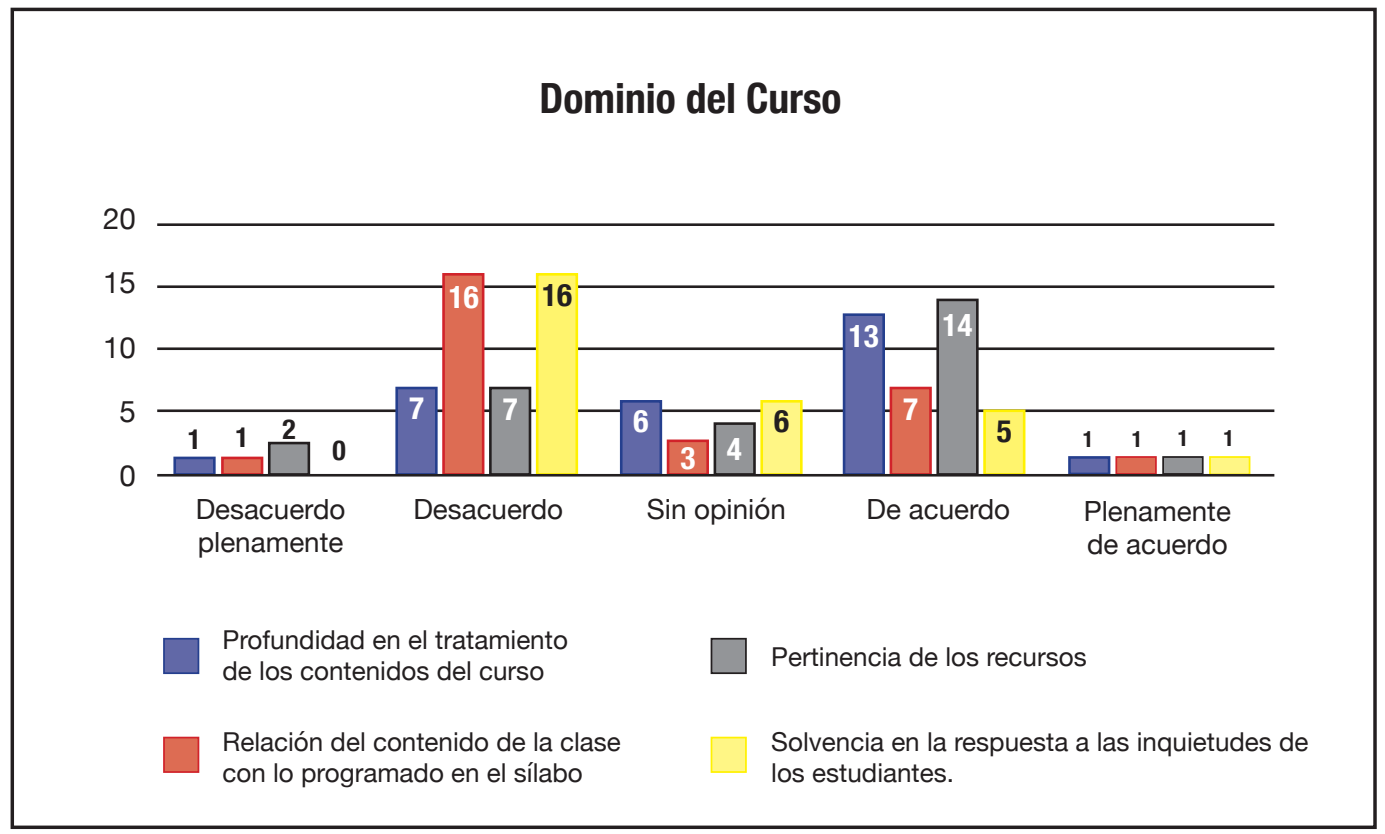

Fuente: Elaboración propia.

La universidad reconoce al docente con el indicador de profundidad en el tratamiento de los contenidos de un curso y el $46.6 \%$ de los docentes encuestados indicó estar de acuerdo sobre sentirse satisfechos.

Para el docente, el desarrollo amplio del tema es importante para brindarle al alumno herramientas y oportunidades de aplicación en contexto.

La universidad reconoce al docente con el indicador de contenido de la clase con la programación del sílabo y el $57.1 \%$ de los docentes encuestados indicó estar en desacuerdo sobre sentirse satisfechos, así como con las respuestas a las inquietudes de los estudiantes.

En tal sentido, el criterio de seguir el contenido programado puede impactar en cómo es percibido por el docente, por lo que se podrían crear criterios más flexibles de aplicación. Además, en algunas circunstancias el recojo de dudas y su resolución puede ser manejado según el docente.

La universidad reconoce al docente con el indicador de pertinencia de los recursos utilizados, y el 50\% de los docentes encuestados indicó estar de acuerdo sobre sentirse satisfechos. El uso de variedad de herramientas y recursos aplicados según la sesión son importantes para la ejecución y desarrollo del tema.

\section{Necesidad de consideración}

El desarrollo de la docencia y formar personas requiere que el docente se perciba como valorado, respetado y desarrollado en todo nivel y que la institución en la que labora y forma parte lo reconozca como elemento valioso en su organización. 


\section{Figura 6}

Resultados sobre la dimensión de consideración

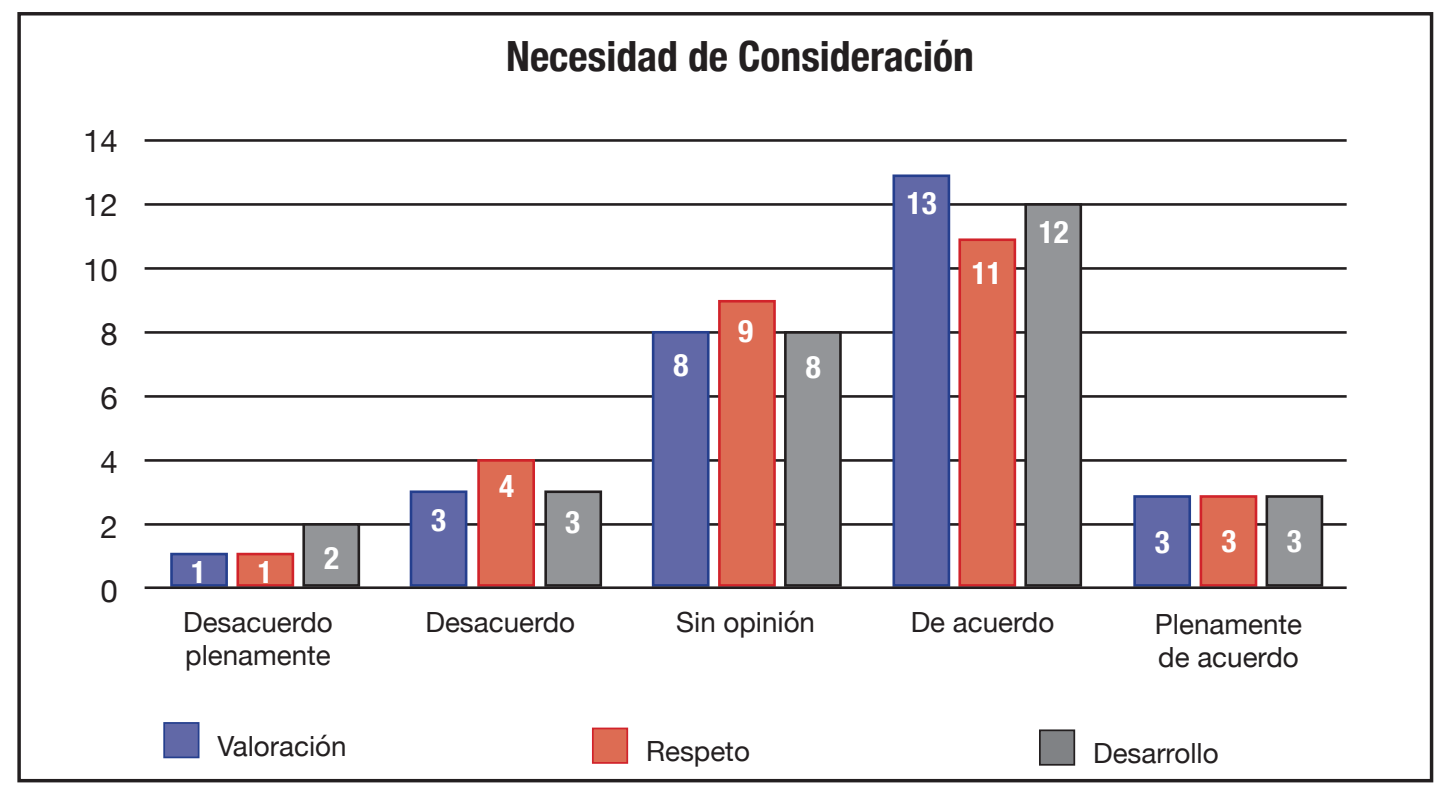

Fuente: Elaboración propia.

La universidad reconoce al docente sobre la dimensión de metodología de enseñanza y dominio del curso, por lo que el $46.4 \%$ de los docentes encuestados indicó estar de acuerdo sobre sentirse valorado. El $39.3 \%$ de los docentes encuestados indicó estar de acuerdo sobre sentirse respetado. El $42.9 \%$ de los docentes encuestados indicó estar de acuerdo sobre sentirse desarrollado a nivel profesional y personal.

El docente puede contrastar la imagen que tiene de sí mismo con lo que la institución espera de él, a fin de reconocer aspectos de mejora y desarrollo a todo nivel.

\section{Opinión de los estudiantes}

Esta dimensión comprende la percepción que el alumno tiene sobre su relación con el docente y sobre el cumplimiento de su responsabilidad, dominio y metodología.

La universidad reconoce al docente con el indicador de puntualidad y conocimiento del estudiante y el $57.1 \%$ de la muestra indicó estar en desacuerdo sobre sentirse satisfechos con la manera como se le reconoce.

Asimismo, la universidad reconoce al docente con el indicador de motivación y expectativas del estudiante. El 50\% de la muestra indicó no estar de acuerdo ni en desacuerdo sobre sentirse satisfechos con la manera cómo se le reconoce.

Las funciones del docente, como mencionan Humberto, Arriola, Henry, Pérez \& Li (2009), puede haber descuidado también la motivación y mediación para la producción del alumno.

Por otro lado, la universidad reconoce al docente con el indicador de dinámicas y participación de estudiantes. Sobre este aspecto, el 46.4\% de la muestra indicó estar en desacuerdo sobre sentirse satisfechos con la manera cómo se le reconoce.

La universidad reconoce al docente con el indicador de evaluación y relación con el estudiante, y el 39.3\% de la muestra indicó estar en desacuerdo sobre sentirse satisfechos con la manera cómo se le reconoce. 


\section{Figura 7}

Resultados sobre la dimensión de opinión de los estudiantes

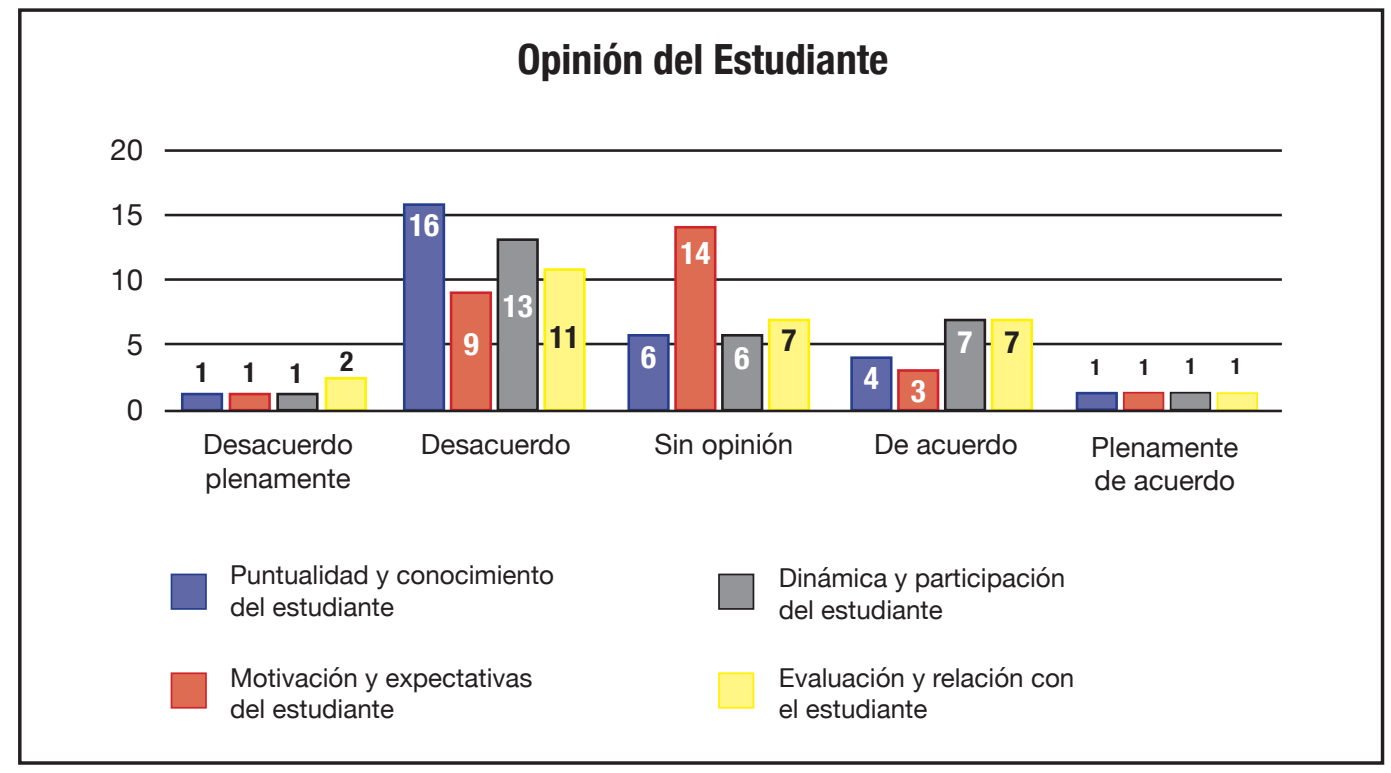

Fuente: Elaboración propia.

Durante el transcurso del semestre académico los estudiantes pueden completar de manera anónima y según su criterio, una encuesta en relación a sus docentes. Según Beltrán (2008), esta percepción puede impactar en como el docente y su desempeño sean percibidos.

No obstante, en una sección de más de 35 alumnos, donde algunos docentes pueden tener hasta dos por día, durante cinco o seis días de la semana, les puede limitar el nivel de personalización del servicio educativo. La relación entre el alumno - docente según otras investigaciones, impacta en la percepción del alumnado.

Del mismo modo, el uso de estrategias y dinámicas de motivación y participación del alumno puede variar según la personalidad del grupo, rasgo de su perfil profesional, nivel de cansancio, estrés, entre otros.

Al mismo tiempo, las estrategias y técnicas de motivación y dinámicas pueden verse limitadas por la personalidad del alumno que impacta también en su nivel de participación. Puede percibirse de manera diferente, el trabajo en equipo o individual, mayor o menor presión por parte del docente, el lenguaje corporal, tono de voz, objetos de la vida real, entre otros. Por tanto, ciertas actividades pueden resultar más atractivas que otras para ciertos alumnos.

También se mencionan algunos estudios sobre las inteligencias múltiples y el desarrollo de habilidades de comunicación según como estas se enfoquen y trabajen de manera alternada. Sin embargo, el uso de ciertas técnicas, podrían no ser maximizadas o valoradas por un grupo de estudiantes. Cabe destacar la amplitud del concepto en relación a las habilidades del docente y características del ambiente de aula.

La encuesta realizada a los alumnos podría, por lo tanto, adaptarse a criterios ampliamente discutidos por un grupo de especialistas, que, a su vez, sea comunicado y sensibilizado al cuerpo de alumnos para recoger la información con mayor data y validez que se requiere.

\section{Necesidad de aprobación}

El docente necesita estar satisfecho en relación a sentir que pertenece a un grupo de trabajo, que 
puede realizar sus funciones de manera correcta y que sus posibles errores pueden ser recogidos eficientemente.

En este sentido, en relación con el sistema de evaluación basado en la opinión de los estudiantes: el $53.6 \%$ de los docentes encuestados indicó estar de acuerdo sobre sentir que pertenecen a una institución. El $50 \%$ de los docentes encuestados indicó estar de acuerdo sobre sentir que realizan su trabajo correctamente. El $46.4 \%$ de los docentes encuestados indicó estar de acuerdo sobre sentir que pueden corregir sus errores.

\section{Figura 8}

\section{Resultados sobre la dimensión de aprobación}

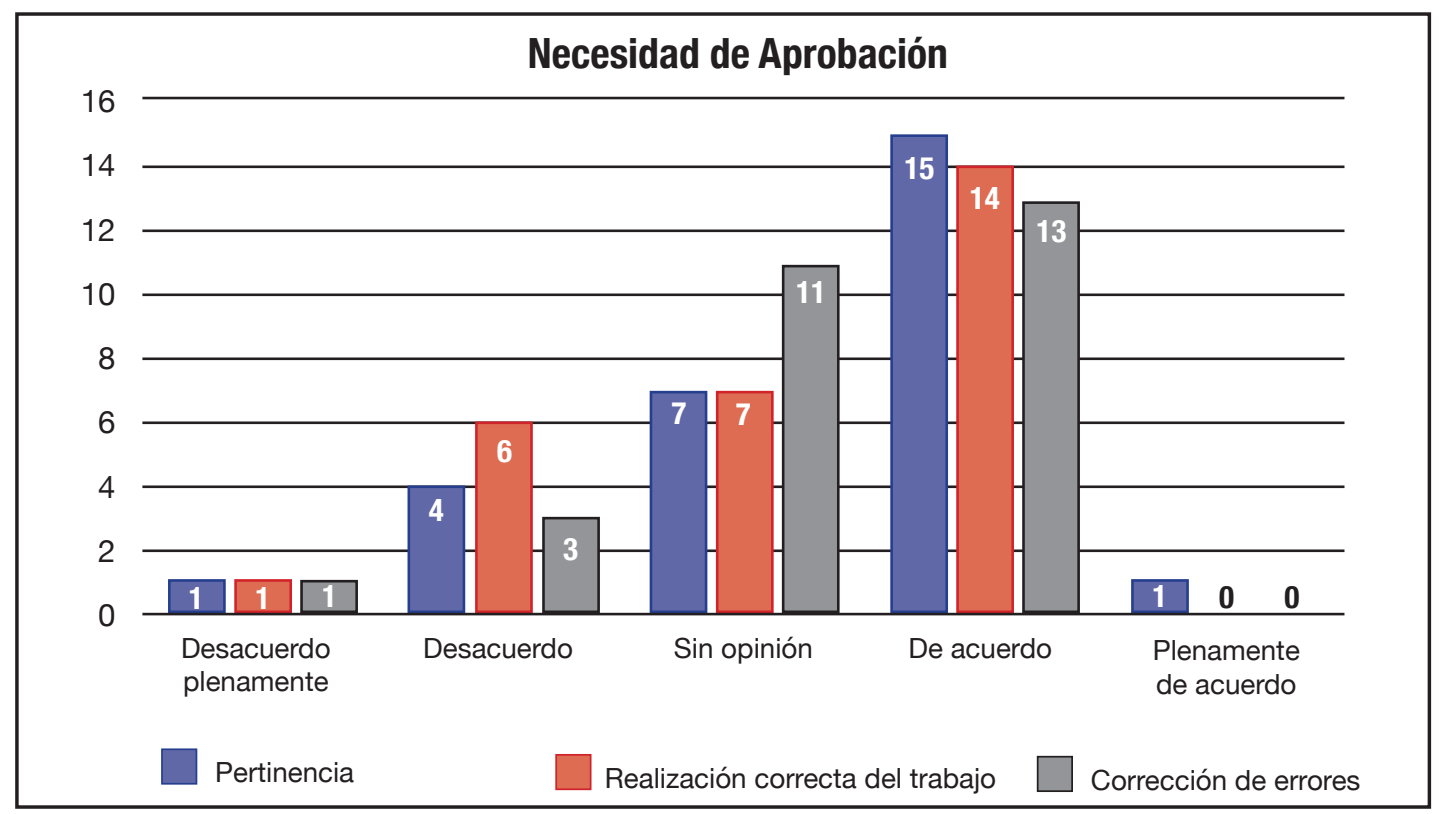

Fuente: Elaboración propia.

Hay una necesidad de permanencia por parte del docente, quien, a su vez, necesita sentirse valorado por sus alumnos. La opinión de ellos impactará en su ejercicio docente. Se debe determinar cómo se realiza la conexión con los estudiantes y cómo impacta en el alumnado, y establecer una priorización de sus impactos en las áreas de conocimiento, como mencionan Domínguez-Lara \& Fernández-Arata (2019).

Al ser un sistema que brinda retroalimentación en el ciclo siguiente de su ejecución, no permite una corrección inmediata o brindar aspectos de mejora oportunos para una corrección en el grupo y momento donde se detecta una falencia.

En cierta medida, la corrección extemporánea puede ser utilizada como mecanismo para reafirmar la práctica docente e incentivo adicional. Por otro lado, el alumno también podría necesitar una retroalimentación emocional para su autopercepción, según Cortés, Campos \& Moreno (2014). Y esto podría también impactar en la evaluación hacia el docente.

Asimismo, hay que considerar que en los criterios de percepción existe un factor emocional que cumple un factor clave para la determinación de aspectos de satisfacción.

\section{Evaluación académica}

El sistema de evaluación utilizado por la universidad comprende la revisión de los exámenes entregados, participación en actividades académicas, uso de la plataforma virtual y actividades de capacitación docente. 
La universidad reconoce al docente con el indicador de entrega de exámenes de calidad y el 50\% de los docentes encuestados indicó estar en desacuerdo sobre sentirse satisfechos con la manera como la universidad le reconoce.

La universidad reconoce al docente con el indicador de participación en actividades académicas y el $46.4 \%$ de los docentes encuestados indicó estar de acuerdo sobre sentirse satisfechos con la manera como la universidad le reconoce.
La universidad reconoce al docente con el indicador de uso de la plataforma virtual y el $46.4 \%$ de los docentes encuestados indicó estar de acuerdo sobre sentirse satisfechos con la manera como la universidad le reconoce.

La universidad reconoce al docente con el indicador de participación en actividades de capacitación docente por calidad educativa y el $50 \%$ de los docentes encuestados indicó estar de acuerdo sobre sentirse satisfechos con la manera como la universidad le reconoce.

\section{Figura 9}

Resultados sobre la dimensión de evaluación académica

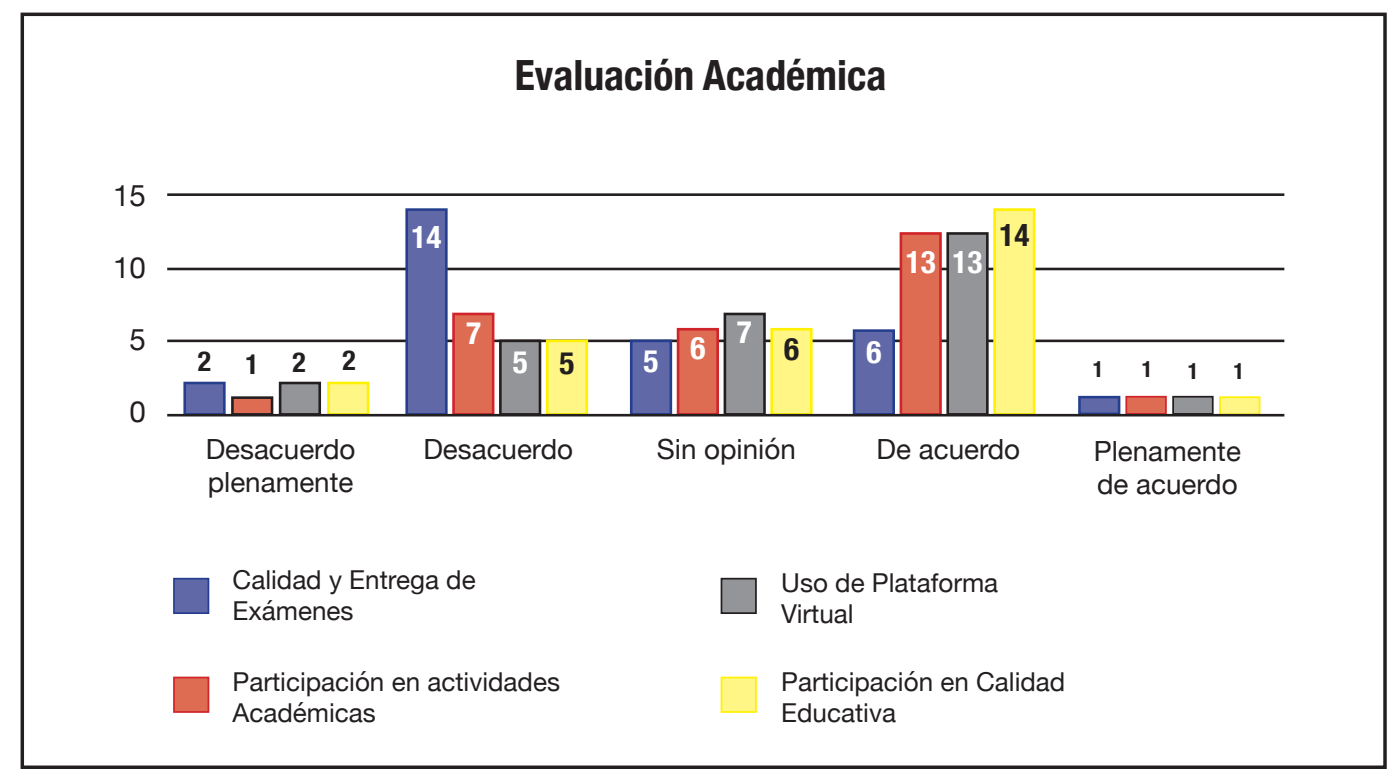

Fuente: Elaboración propia.

En tal sentido, los criterios utilizados por el departamento académico correspondiente para la revisión de los exámenes quedan hasta cierto punto a subjetividad de este, impactando en la percepción del docente.

Los eventos realizados por la dirección correspondiente, que incluyen aspectos de integración, culturales, entre otros, también impactan en la realización de su trabajo.

El indicador de cotejo sobre el uso de la plataforma virtual varía desde el tiempo de conexión, hasta las herramientas y el porcentaje de participación de los estudiantes.

La evaluación indicada por el departamento de calidad educativa, según el curso del programa de entrenamiento y capacitación docente, forma parte de este ítem de evaluación.

\section{Necesidad de reputación}

El docente necesita estar satisfecho en relación a sentir que destaca en el grupo que forma parte, que es admirado por sus colegas, y que es recompensado. 
En relación al sistema de evaluación basado en el sistema de evaluación académica: el $46.4 \%$ de los docentes encuestados indicó estar de acuerdo sobre sentir que destacan en el grupo. El 37.5\% de los docentes encuestados indicó estar en desacuerdo sobre sentir que son admirados en el grupo. El 42.9\% de los docentes encuestados indicó estar de acuerdo sobre sentir que son recompensados.

\section{Figura 10}

Resultados sobre la dimensión de reputación

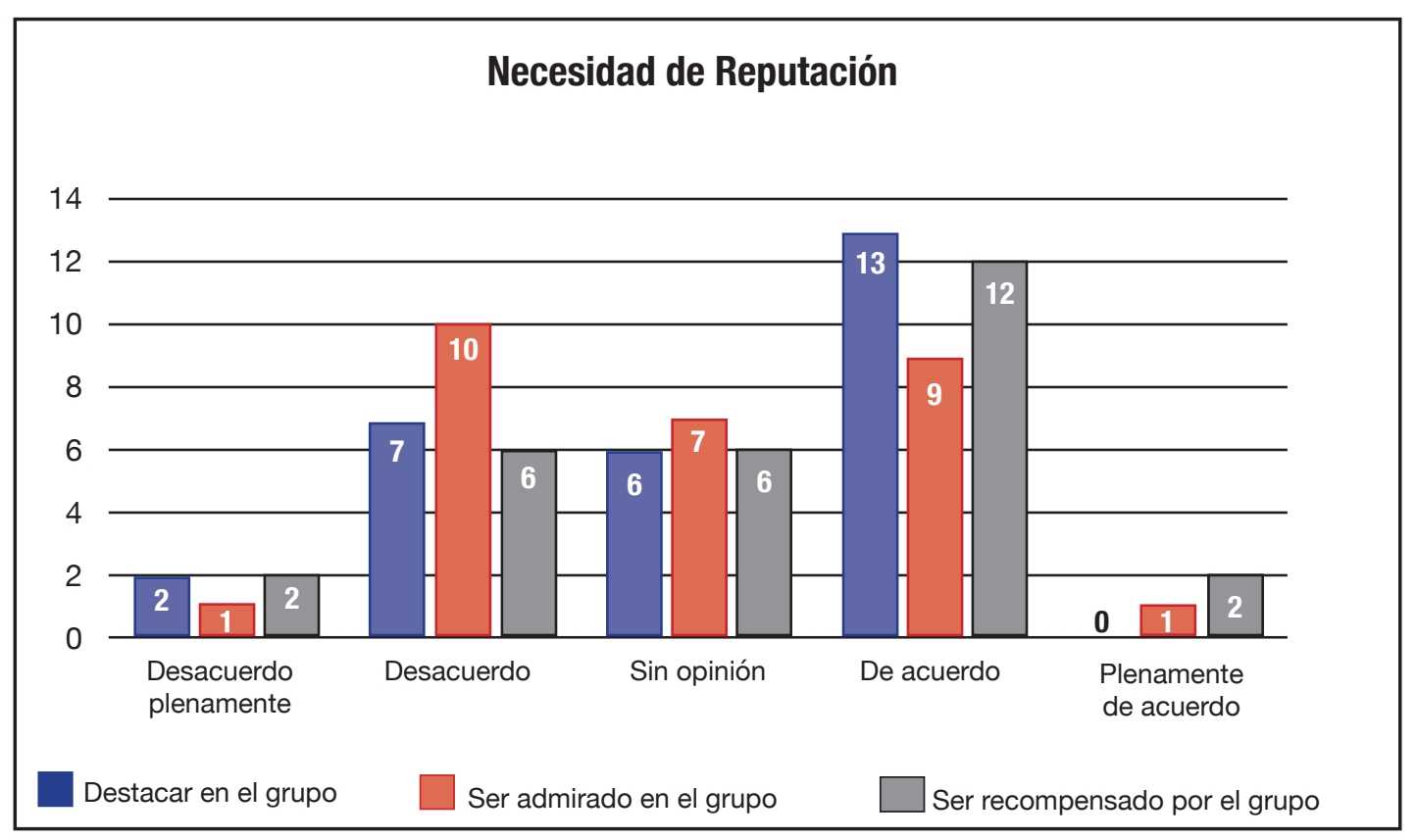

Fuente: Elaboración propia.

La necesidad de destacar en el docente puede variar según su autopercepción, y en consecuencia de la evaluación, poder sentir que destaca por su desenvolvimiento y cualidades que presenta.

Es importante poder incluir dentro del proceso una autoevaluación inicial para reconocer sus características y aspectos de mejora, como mencionan Milicic, et al. (2008).

Como ejemplo, la dirección de inglés valora las iniciativas personales de los docentes a través de actividades de integración.

Además, el ocupar una posición alta en la escala de puntuación del docente lo coloca en una posición diferenciada ante los demás, y la posibilidad de sentirse admirados y recompensados por la institución y grupo de referencia.

\section{Discusión de los resultados}

Primero, se determina que la relación entre ambas variables se impulsa por un componente motivacional extrínseco a través de una compensación monetaria. Este tiene un impacto positivo en la satisfacción del docente, sobre su autoconfianza, consideración, aprobación y reputación. No obstante, se recomienda a la institución ampliar el catálogo de compensación; es decir, ofrecer otros incentivos que puedan ser seleccionados por el docente a manera 
de "paquetes" para personalizar sus deseos y necesidades. De manera complementaria, se puede realizar una investigación de preferencias, como mencionan G. Martínez-Chairez y A. Guevara-Araiza (2015).

El impacto emocional y físico repercute en el proceso, como menciona Urriola (2013), por lo que se recomienda una actualización periódica y que, a su vez, integre y fomente el trabajo en equipo y que, asimismo, se traduzca en resultados positivos.

Debe haber una retroalimentación técnica, académica y política, como mencionan Lora \& Reyes Chávez (2008) y revisión de los indicadores, para que la información procesada siga siendo de calidad y también formal, según estándares de exigencia y como soporte para la planificación y gestión educativa, dentro de un modelo de integración y eficiencia como menciona Isoré (2010).

Segundo, el sistema de evaluación basado en responsabilidad con la satisfacción de la necesidad de autoconfianza, se determina por la administración institucional y enfatiza el porcentaje de cumplimiento del trabajo realizado, en la asistencia, registro de notas y elección de delegado. Se realiza de manera sistematizada e impacta en su autoconfianza. Se concluye que el impulso a definir su existencia en la institución lo orienta a la ejecución de sus tareas, como señala C. Danielson (2011) y la mayoría está de acuerdo con la satisfacción de su competencia, autonomía y consciencia de su fortaleza.

Se ha recomendado que los indicadores reciban un tratamiento y control orientado a la funcionalidad y eficiencia mediante un consenso, y de amplio conocimiento, a fin de evitar medidas correctivas que puedan afectarlos. Se podría limitar los beneficios de la institución si no se aplican criterios de funcionalidad y eficiencia.

Tercero, el sistema de evaluación basado en la metodología y dominio del curso con la satisfacción de la necesidad de consideración, se determina con el cumplimiento de criterios metodológicos del curso validados a través de una observación de una clase grabada, orientada a mejorar la calidad de manera continua, como mencionan Gómez y Valdés (2019).

La necesidad de consideración del docente está basada en el impulso de influencia para con los demás y el reconocimiento de su esfuerzo. La mayoría se siente satisfecha al ser valorada, respetada y desarrollada por la forma como enseña la materia que domina. Se ha recomendado el acompañamiento de habilidades y complementación docente a través de fuentes electrónicas y presenciales para acceder en su tiempo libre y seguir mejorando a nivel profesional y personal. Estas herramientas podrán ser aprovechadas según el interés y perfil de cada docente, como menciona Sánchez (2012).

Cuarto, la evaluación a través de la opinión de los estudiantes y la necesidad de aprobación, está determinada por una serie de preguntas que delimitan diversos aspectos contemplados sobre la percepción del docente. Esta necesidad se impulsa por el crecimiento de este.

Se ha determinado que la mayoría se siente satisfecha al poder perfeccionar habilidades junto a la organización y percepción de aspectos de mejora. Se ha recomendado que se impartan campañas de conocimiento del clima y cultura institucional y compromiso del alumno hacia una imparcialidad y valoración del aspecto formativo de la evaluación.

Para que el docente pueda ser más empático y desarrollar la escucha activa como menciona Márquez (2016), el impacto en el desarrollo competencial del docente mejorará la relación entre ambas partes, por ser una necesidad de entrenamiento docente como señala Marín, et al. (2004), así como la importancia de la participación objetiva.

Quinto, la evaluación basada en el desenvolvimiento académico y la necesidad de 
reputación, se determina por la conducta del docente en base a resultados y la percepción entre colegas. Se basa en el impulso de lograr crecimiento. La mayoría se siente satisfecho al destacar, ser admirado y recompensado por la institución. Como señala Barranco (2000), los colaboradores de la empresa se conectan con la institución y sus objetivos al ser tratados como miembros partícipes y destacando su importancia constante, y ampliarlo a un autoconcepto donde pueda ser consciente de qué debe hacer para ser mejor y más valioso para la institución, maximizando el valor para ambas partes.

\section{REFERENCIAS}

Alderfer, C. (1969). An empirical test of a new theory of human needs. Organizational behavior \& human performance. 142-175.

Beltrán, M. R. (2008). The Evaluation of Teacher Perfomance at the University. Revista Electrónica de Investigación Educativa. Recuperado de: http://redie.uabc. $\mathrm{mx} /$ NumEsp1/contenido-rueda.html

Barranco, F. (2000). Marketing interno y gestión de recursos humanos. Madrid, España: Pirámide.

Berry-Leonard, L. (1981). The employee as customer. Chicago, Estados Unidos: American Marketing Association.

Cortés, E., Campos, M. \& Moreno, M. P. (2014). Priorización de las Dimensiones de Evaluación al Desempeño Docente por el Estudiante, en tres Áreas del Conocimiento. Formación Universitaria, 10(2), 3-10. Recuperado de: https://doi.org/10.4067/ S0718-50062014000200002

Danielson, C. (2011). Competencias docentes: desarrollo, apoyo y evaluación. Programa de Promoción de la Reforma Educativa en América Latina y el Caribe. 5-18.

Dominguez-Lara, S. \& Fernández-Arata, M. (2019). Autoeficacia académica en estudiantes de Psicología de una universidad deLima. RevistaElectrónica de Investigación Educativa, 21(1), 1. Recuperado de: https:// doi.org/10.24320/redie.2019.21.e32.2014
García Cabrero, B., Loredo Enríquez, J. \& Carranza Peña, G. (2008). Análisis de la práctica educativa de los docentes: pensamiento, interacción y reflexión. Analysis of the Teacher's Educational Practice: Didactic Thinking, Interaction and Reflection. Revista Electrónica de Investigación Educativa. Recuperado de: http://redie.uabc.mx/NumEsp1/contenidogarcialoredocarranza.html

Gómez, L. F. \& Valdés, M. G. (2019). La evaluación del desempeño docente en la educación superior. 7, 479-515. Recuperado de: https://doi.org/10.20511/pyr2019.v7n2. 255

Hernández, R., Fernández, C. y Baptista, P. (2014). Metodología de la investigación. México: McGraw-Hill.

Hortigüela, D., Ausín, V., Delgado, V. \& Abella, V. (2017). Análisis de la importancia de los criterios de evaluación y el reconocimiento académico docente universitario como indicadores de la calidad educativa en España. Revista de la Educación Superior, 46(181), 75-87. Recuperado de: https://doi. org/10.1016/j.resu.2016.10.002

Humberto, G., Ariola, A., Henry, I., Pérez, A. \& Ii, L. (2009). Evaluación de la función docente según el desempeño de los profesores y la opinión estudiantil. Evaluation of the teaching function related to the professor performance and the student criterion. En Educación Médica Superior (Vol. 23). 
Isoré, M. (2010). Evaluación docente: prácticas vigentes en los países de la OCDE y una revisión de la literatura. París: Preal.

Lora, L. E. \& Reyes, R. (2008). Elementos clave para la evaluación del desempeño de los docentes. Key Elements for the Evaluation of the Teaching Performance. Revista Electrónica de Investigación Educativa. Recuperado de: http://redie.uabc.mx/ NumEsp1/contenido-elizaldereyes.html

Loureiro, S. \& Míguez, M. (2016). Desempeño docente en la enseñanza universitaria: análisis de las opiniones estudiantiles. Cuadernos de Investigación Educativa, 7, 55-67. Recuperado de: https://doi. org/10.18861/cied.2016.7.1.2576

Maslow, A. H. (1991). Motivación y personalidad. Madrid: Díaz de Santos S. A.

Marín, M., María, S., Melero, P. T., Facultad, S., Camilo, P. \& Cela, J. (2004). The training of University Teachers: Needs and Demands from their students. La formación del docente universitario: Necesidades $y$ demandas desde su alumnado. 18(2), 137-151.

Márquez, A. (2016). Editorial: ¿Qué podemos esperar de la evaluación docente? Perfiles Educativos, 1-11.

Martinez-Chairez, G. \&. Guevara-Araiza, A. (2015). La evaluación del desempeño docente. Ra Ximhai, 113-124.

McClelland, D. (1961). The achieving society. New Jersey: Van Nostrand.

Milicic, N., Rosas, R., Scharager, J., García, R., Godoy, C. \& García, M. R. (2008). Diseño, Construcción y Evaluación de una Pauta de Observación de Videos para Evaluar Calidad del Desempeño Docente. Design, Construction, and Assessment of a Guideline for the
Observation of Videos to Evaluate Quality of Teacher's Performance. PSYKHE, 17, 79-90.

Obreque, A., Hernández-Mosqueira, C., PeñaTroncoso, S.,Agredo, M.A. T., Salvatierra,M. O. \& Cáceres-Ramos, B. (2019). Evaluation of teacher performance in Chile: Perception of poorly evaluated teachers. Cuadernos de Pesquisa, 49(172), 144-163. Recuperado de: https://doi.org/10.1590/198053145792

Piña, R. (2010). El desempeño docente y su relación con las habilidades del estudiante y el rendimiento académico en la universidad particular de Iquitos. (Tesis doctoral). Iquitos, Perú: Universidad Nacional Mayor de San Marcos.

Ruiz, M. (2012). La selección docente. Piedra angular para crear un sistema educativo de calidad. REIFOP, 187-194.

Santos Del Real, A. (2012). Evaluación docente. Teacher evaluation. Recuperado de: www. sep.gob.mx/es/sep1/C0570511

Sánchez, J. (2012). Gestión del talento del personal universitario. Algunas reflexiones y propuestas. Ciencia y Sociedad, 95-121.

Urriola, K. (2013). Sistema de evaluación del desempeño profesional docente aplicado en Chile. Percepciones y vivencias de los implicados en el proceso. El caso de la ciudad de Concepción. (Tesis doctoral). BarcelonaEspaña: Universidad de Barcelona.

Visbal-Cadavid, D., Mendoza-Mendoza, A. \& Corredor-Carrascal, K. (2015). Evaluación del desempeño docente mediante el análisis envolvente de datos: un estudio de caso. Evaluation of the teacher performance using data envelopment analysis: a case study. 11(2). Recuperado de: https://doi. org/10.18041/entramado.2015v11n2.22216

Fecha de presentación: 17-02-20.

Fecha de aceptación: 20-07-20. 